\title{
LA CONSERVACIÓN DE LA FITODIVERSIDAD ECOSISTÉMICA EN LA SIERRA MAESTRA, CUBA ORIENTAL
}

\begin{abstract}
Orlando J. Reyes', Luz Margarita Figueredo Cardona ${ }^{1}$
1 Centro Oriental de Ecosistemas y Biodiversidad (BIOECO), Ministerio de Ciencia, Tecnología y Medio Ambiente (CITMA), José A. Saco No. 601, esq. Barnada, Santiago de Cuba, Cuba. E mail joel@bioeco.cu

RESUMEN

El establecimiento de los ecosistemas prioritarios para la conservación de la fitodiversidad del macizo montañoso Sierra Maestra constituye el principal objetivo de esta investigación. Aplicando la metodología de la Escuela Zurich Montpelier se realiza un estudio fitocenológico de las asociaciones de la Sierra Maestra. Mediante la evaluación naturalística (criterios fundamentales y complementarios), la viabilidad y los criterios de los Inventarios Biológicos Rápidos se determinaron las formaciones vegetales que se proponen como objetos de conservación y las zonas que deben priorizarse respecto a las medidas conservativas. Se considera que la Sierra Maestra debe constituirse en un "Área de conservación funcional" u "Objeto de conservación de filtro grueso" y/o "Nacional o Gruesa"; concentrando los esfuerzos de manejo y protección en la parte baja, declarando a su vez las áreas por encima de los $800 \mathrm{~m} \mathrm{snm}$ como una zona de "valor para la conservación de las aguas, los suelos y la biodiversidad".
\end{abstract}

Palabras clave: Ecosistemas, fitodiversidad, conservación, Sierra Maestra, Cuba.

Recibido: 17 de Abril de 2019. Aceptado: 04 de Diciembre de 2019.

Received: April 17, 2019. Accepted: December 04, 2019.

\section{SIERRA MAESTRA'S PHYTOECOSYSTEM CONSERVATION, EASTERN CUBA}

\begin{abstract}
The establishment of priority ecosystems for the conservation of the phytodiversity of the Sierra Maestra mountain range is the main objective of this research. A phytocenological study of the associations of the Sierra Maestra is presented, applying the methodology of the Zurich Montpelier School. By means of naturalistic evaluation (fundamental and complementary criteria), the viability and criteria of the Rapid Biological Inventories were determined the vegetal formations that are considered as objects of conservation and the zones that must be prioritized with respect to the conservative measures. It is considered that the Sierra Maestra should constitute a "functional conservation area" and "coarse filter conservation object" and/or "National or Coarse"; concentrating management and protection efforts in the lower part, declaring the areas above $800 \mathrm{~m}$ over sea as an area of value for the conservation of water, soils and biodiversity.
\end{abstract}

Key words: Ecosystems, phytodiversity, conservation, Sierra Maestra, Cuba.

Cómo citar este artículo: $O$. Reyes, L. Figueredo. "La conservación de la fitodiversidad ecosistémica en la Sierra Maestra, Cuba oriental.", Revista Politécnica, vol. 15 no.30 pp.105-118, 2019. DOI: 10.33571/rpolitec.v15n30a10 


\section{INTRODUCCIÓN}

En la Sierra Maestra se han realizado esfuerzos para la conservación de la fitodiversidad con diferentes criterios. Inicialmente se creó el Gran Parque Nacional Sierra Maestra con objetivos conservacionistas y se definieron un gran número de reservas naturales con principios principalmente florísticos, las que separaban las áreas de mayor interés conservacionista. Algunas de estas reservas fueron posteriormente estudiándose y redelimitándose en sus áreas más conservadas. En 1999 se promulgó el Decreto Ley 201 en que se creó el actual Sistema Nacional de Áreas Protegidas (SNAP) lo que modificó lo anteriormente expuesto y que rige en la actualidad.

En la Sierra Maestra, reconocida por el Sistema Nacional de Áreas Protegidas como Región Especial de Desarrollo Sostenible, hay: tres parques nacionales (Desembarco del Granma, Turquino y La Bayamesa), seis reservas ecológicas (Caraquitas, Caracas, El Gigante, Pico Mogote, Siboney-Juticí y Hatibonico), cuatro reservas florísticas manejadas (El Macío, Loma del Gato-Monte Libano, Monte Bisse y Pozo Prieto), una reserva natural (El Retiro), un elemento natural destacado (Salto de Alcarraza), un paisaje natural protegido (Gran Piedra) y dos áreas protegidas de recursos manejados (Baconao y La Tabla).

Como el estado cubano promueve un activo proceso de recuperación y protección de los recursos naturales [1], el aumentar sistemáticamente los conocimientos de la complejidad de los elementos de la Sierra Maestra, de su potencial pasado y actual, de su capacidad de carga y de su resiliencia, es muy importante para garantizar el desarrollo sostenible de nuestra nación, pues la existencia de muchas ciudades colindantes y de su población autóctona depende de la conservación de su potencial acuífero, de sus suelos y de su biodiversidad.

Loidi [2, 3, 4] consideró que la "evaluación naturalística" aporta criterios de la mayor trascendencia para organizar la gestión de un territorio con intención de conservar y beneficiar lo natural y lo biodiverso, de mantener la funcionalidad de los sistemas y de impulsar su uso sostenible. Reyes [5] expuso que en nuestro archipiélago es valido aplicar, con adaptaciones (incluso locales), este punto de vista y que conjugado con otros principios $[6,7,8,9,10,11$, 12] es posible tener criterios para la conservación de los ecosistemas cubanos.

Por ello, el objetivo de este trabajo es reconocer los ecosistemas de la Sierra Maestra que de acuerdo a su estado actual y a los criterios metodológicos utilizados deben priorizarse con vistas a la conservación de la fitodiversidad.

\section{MATERIALES Y MÉTODOS}

En este trabajo, se considera la conservación ecosistémica como fundamental. En el ecosistema se encuentran las especies, incluso las endémicas, amenazadas, entre otras; es además donde se produce la alteración y los efectos naturales y antrópicos. Cuando se preserva el ecosistema se conservan también las especies y las condiciones que permiten su evolución y perpetuación; se mantiene además el desarrollo y funcionamiento de los sistemas.

Se opina igualmente, que las fitocenosis (syntaxa, asociaciones), que son producto de la evolución biológica en el sitio bajo las condiciones ecológicas respectivas, se determinan generalmente de varias muestras en cada tipo, donde se estudian la composición florística, la abundancia dominancia de las especies, la estructura y las condiciones ecológicas que las caracterizan. Similarmente, se tipifican los elementos que tienen el mayor influjo en el funcionamiento de dichos ecosistemas por el aporte de sus detritos y que intervienen además en el flujo energético que penetra al interior de la cobertura y por tanto en el tipo de especies que crecen en su interior. Por ello, se considera que las fitocenosis pueden utilizarse convenientemente como una representación válida de dichos ecosistemas.

La evaluación naturalística consiste en un conjunto de criterios básicos, cada uno de los cuales debe ser procesado y ponderado en relación con los demás. La combinación de todos conformará la valoración conjunta. Loidi [2, 3] elaboró como "criterios fundamentales", que son los que tratan de interpretar las propiedades intrínsecas de las comunidades vegetales: naturalidad $(N)$, resiliencia $(R)$, amenaza $(T)$, valor florístico - fitocenótico $(F)$ y rareza $(R)$ y como "criterios complementarios", que representan aspectos particularmente relevantes de los 
servicios que las formaciones vegetales prestan a los ecosistemas y a las sociedades humanas, y que son: retención de carbono (RC), protección del suelo (S), mejoramiento y mejora de la calidad de las aguas $(\mathrm{H})$ y el coeficiente de urgencia territorial para la protección del ecosistema (E).

En nuestras condiciones de vegetación tropical y de acuerdo a los análisis realizados a múltiples ecosistemas cubanos [5, 13], se modifican las anteriores propuestas de Loidi, considerándose parte de los "criterios fundamentales": naturalidad $(N)$, resiliencia $(R)$, valor florístico - fitocenótico $(F)$ y unicidad fitocenótica 0 territorialidad fitogeográfica (UT), las que de conjunto constituyen el "valor biológico" (B). Las amenazas al ecosistema (A), son siempre negativas a las fitocenosis, reduciendo, afectando $y / 0$ minimizando a su vez la integridad de su "valor biológico" (B), por lo cual se restan del mismo. Los criterios complementarios y la valoración final (IC) también se modifican como se expone más adelante.

La Sierra Maestra presenta típicas condiciones tropicales, las que debido a sus valores de temperatura y precipitación inducen diferencias en los procesos de meteorización del suelo y en el desarrollo de la vegetación; tiene además disimilitudes altitudinales importantes que provocan una substancial diversidad cliserial, igualmente está fuertemente antropizada y con diversos estadios cenogenéticos, por eso es válida la adaptación de los criterios anteriormente expuestos.

\section{Criterios fundamentales}

Naturalidad (N)

Como naturalidad se evalúa el grado de aproximación a la vegetación potencial de la zona estudiada. Se considera como máximo de naturalidad cuanto más próxima está de dicha vegetación potencial. En el caso de fitocenosis secundarias, el valor se aumenta en el sentido siguiente: comunidades secundarias inmediatas, comunidades tempranas (Fiera I y Homeostasis I), comunidades tardías (Fiera II y Homeostasis II) y climax o etapa madura.

0 - área urbanizada y/o en cultivo; 1- vegetación ruderal y/o de campos cultivados abandonados, comunidades secundarias inmediatas, potreros de camagueyana; 2- vegetación pionera, Fiera I; 3plantaciones forestales de especies exóticas; 4- pastizales y potreros en uso de yerba de guinea; 5- matorrales secundarios desarrollados, Homeostasis I, matorral y/o herbazal de galería; 6vegetación de áreas húmedas, presas y del borde de las mismas; 7- bosques jóvenes naturales, Fiera II adelantada; 8- bosques naturales medianamente a poco alterados (incluso bosques de galería), Homeostasis II; 9- cualquier tipo de vegetación potencial natural, herbazal de humedal natural, herbazal halófito natural, manglares achaparrados; 10- bosques maduros y/o poco alterados estructuralmente, manglares desarrollados, matorrales climácicos, vegetación de dunas costeras, sabanas naturales.

Resiliencia (R)

Capacidad de los sistemas para enfrentar tensiones y de recuperarse cuando dichas tensiones disminuyen [14], es decir, la capacidad intrínseca del tipo de vegetación.

0 - área sin vegetación, urbanizada y/o en cultivo; 1- comunidades secundarias inmediatas sin dominancia de helechos; 2- vegetación pionera perenne con dominancia de helechos; 3pastizales secundarios perennes y/o plantaciones forestales de especies exóticas; 4- matorrales secundarios consolidados, Homeostasis I; 5bosques arbustosos en sucesión adelantada, Fiera II; 6- vegetación halófita costera, manglares achaparrados y herbazal halófito, vegetación de humedal en correspondencia con el ecótopo; 7 bosques naturales medianamente alterados, Homeostasis II; 8- vegetación potencial, bosques maduros $\mathrm{y} / \mathrm{o}$ poco alterados estructuralmente, manglares desarrollados, matorrales climácicos, vegetación sobre las dunas costeras.

Amenazas al ecosistema $(A)$

La Sierra Maestra tiene áreas protegidas (parques nacionales, reservas ecológicas, reservas florísticas manejadas, entre otras), en que la función fundamental de esas categorías es la conservación de esos ecosistemas, tienen además planes operativos y/o de manejo, así como personas dedicadas a ese objetivo (protección y manejo). En la Sierra Maestra los territorios por encima de los $800 \mathrm{~m}$ snm (sobre todo por encima de $1200 \mathrm{~m} \mathrm{snm}$ ) quedaron prácticamente despoblados después de la migración de los años 60 del Siglo XX; a su vez, presentan una topografía muy accidentada y están muy alejadas de los poblados y otras concentraciones humanas, por ello están más 
conservados y las amenazas de la intervención del hombre se minimizan.

Debido a lo antes expuesto, los ecosistemas comprendidos en este grupo de áreas protegidas y por encima de $800 \mathrm{~m}$ snm, no se les considerarán amenazas (se prescinde de las naturales) y por ello se le dará el valor mínimo.

Hay además en esta sierra zonas agropecuarias con campesinos u otro personal que explotan los ecosistemas con diversos fines (cultivos, crianza de ganado, obtención de madera, entre otros); por ello, en las fitocenosis en estas áreas se estimarán las amenazas (mayor valor a medida que la fitocenosis está más amenazada) a que están sometidas dichas comunidades vegetales.

1- bosques y matorrales naturales climácicos por encima de los $1500 \mathrm{~m}$ snm; 2- bosques, matorrales $\mathrm{y} / \mathrm{o}$ herbazales naturales maduros 0 secundarios por encima de los $800 \mathrm{~m}$ snm, o sobre lapiez 0 diente de perro; 3 - bosque arbustivo secundario premontano; 4- vegetación halófita costera; 5- pastizales secundarios, matorrales, herbazal de humedal, vegetación alrededor de lagunas, en la parte baja; 6manglares y comunidades asociadas; 7 - bosques, matorrales y herbazales de la parte llana y premontana baja, vegetación sobre las dunas costeras (debido al turismo).

Valor florístico - fitocenótico (F)

El "valor florístico-fitocenótico" está dado por las especies (endémicas, amenazadas, entre otras) componentes de la fitocenosis y su tipo, su naturalidad, su grado de complejidad estructural y diversidad, su endemicidad y valor regional, así como su significación biogeográfica e histórica.

0 - área sin vegetación y/o en cultivos; 1 vegetación pionera de zonas bajas; 2- pastizales secundarios perennes y plantaciones forestales con especies exóticas, vegetación halófita costera; 3- matorrales secundarios, manglares uniespecíficos; 4- bosques arbustosos secundarios, Homeostasis I, comunidades asociadas al manglar, vegetación de las dunas costeras; 5- fitocenosis interiores de los manglares relativamente ricas en especies, vegetación de humedal en correspondencia con el ecótopo; 6- herbazal de humedal endémico; 7bosques medianamente alterados; 8 - bosques maduros poco alterados estructuralmente, bosques secundarios ricos en especies,
Homeostasis II; 9- pluvisilvas poco alteradas, bosques y matorrales nublados, bosques y matorrales naturales costeros conservados.

Unicidad fitocenótica 0 Territorialidad fitogeográfica (UT)

En lugar de la rareza $(R)$, se considera la unicidad (singularidad) de las fitocenosis. Es decir, si dichas fitocenosis se desarrollan solo en este macizo o están ampliamente distribuidas, si tienen sus equivalentes en otro lugar; además, se tiene en cuenta su extensión y los que presentan una posición geográfica particular.

0- fitocenosis ampliamente distribuida; 1presencia extensa en otros territorios y pequeña en la Sierra Maestra; 2- pequeña extensión tanto en el macizo como fuera del mismo; 3comunidades presentes fuera del macizo, con cinco (5) o menos muestras en el mismo; 4fitocenosis únicas de esta sierra ampliamente distribuidas; 5- comunidades únicas del macizo en cayos aislados relativamente extensos, 6fitocenosis única encontrada en pequeños cayos aislados, 7- única y hallada en un solo lugar de la sierra.

El "valor biológico" (B) de la fitocenosis se determina mediante la suma de estos cinco parámetros: $\mathrm{B}=(\mathrm{N}+\mathrm{R}+\mathrm{F}+\mathrm{UT})-\mathrm{A}$.

Criterios complementarios

Retención de carbono ( $R C)$

En este aspecto se estima la función de la vegetación en la retención del carbono por el aparato fotosintético, cantidad que se considera aumente principalmente con su biomasa.

1.0- comunidades herbáceas, pastizales, vegetación halófita, campos cultivados abandonados; 1.2- herbazal de humedal, herbazal de galería, 1.4- matorrales y bosquetes naturales, manglares achaparrados, Homeostasis I; 1.6 plantaciones forestales desarrolladas o bosques naturales juveniles, Fiera II; 2.0 - manglares densos, bosques maduros o poco alterados estructuralmente, Homeostasis II.

Protección del suelo y mantenimiento de la calidad de las aguas (S-H)

Respecto a la protección del suelo, se trata de estimar la capacidad de la vegetación para retener suelo, principalmente por medio de un sistema radical capaz de mantener nutrientes y evitar el 
transporte del suelo por las precipitaciones. Con relación a la función de la vegetación en la regulación del régimen hidrológico de la zona (protección de los recursos hídricos) y al mantenimiento de la calidad del agua (autodepuración, o sea valor hidrológico como capacidad de depuración de las aguas).

En las condiciones de la Sierra Maestra, debido a que la mayor parte de su territorio tiene una topografía muy escabrosa y a que los suelos son fácilmente erosionables, estos criterios tienen primordial importancia y se interrelacionan entre si, por lo que deben unirse y ponderarse adecuadamente. A su vez, ambos dependen fundamentalmente de la diversidad y densidad de cobertura de los estratos de la vegetación, de la capacidad para retener las partículas de suelo en suspensión, de limitar la velocidad de la escorrentía y de propiciar la infiltración de las aguas.

0 - áreas sin vegetación, 1 - vegetación halófita, de saladares, cultivos poco densos; 2 - herbazal de humedal, potreros de camagueyana, manglares achaparrados; 4 - matorrales de diversos tipos, Homeostasis I en la parte baja, pastizales de yerba de guinea; 5 - plantaciones juveniles, herbazal de galería; 7 - bosques naturales juveniles o medianamente degradados, Homeostasis I en la pluvisilva; 9 - bosques maduros $y / 0$ poco alterados, plantaciones forestales con los estratos arbóreo, herbáceo y arbustivo densos, manglares desarrollados, herbazales densos constituidos por helechos.

Se considera que el "coeficiente de urgencia territorial para la protección del ecosistema" no debe valorarse en este macizo, ya que la densidad de población es muy diversa, localmente concentrada y/o dispersa, por lo que la valoración de este parámetro es muy difícil e inexacta.

Interés para la conservación (IC)

Loidi [3, 4] consideró el interés para la conservación (IC), como la estimación final y que dará un valor determinado para la fitocenosis o el área tratada y se obtiene multiplicando B por los demás valores estimados en los criterios complementarios $(R C, S, H)$, o sea $I C=B \times R C \times$ $\mathrm{S} \times \mathrm{H}$.

En este estudio se utiliza la sumatoria, en lugar de la multiplicación, de los criterios fundamentales y complementarios, puesto que se observó que se producían grandes diferencias entre ecosistemas parecidos debido a una disimilitud en un criterio complementario, es decir, $\mathrm{IC}=\mathrm{B}+\mathrm{RC}+\mathrm{S}-\mathrm{H}$.

Viabilidad

Importante en la valoración de un ecosistema es la "viabilidad" [6, 7]. En este contexto se refiere a la habilidad de una comunidad / sistema ecológico de persistir durante un período específico de tiempo. Según estos autores la "viabilidad" depende del tamaño, condición y contexto paisajístico. El primero es el área requerida para asegurar la supervivencia, recolonización, sucesión y tolerar los disturbios ("área dinámica mínima"); la "condición" es una medida integral de la calidad de los factores bióticos y abióticos, las estructuras y los procesos que definen a los objetos de conservación. A su vez, el "contexto paisajístico" representa la integridad de los procesos ecológicos y regímenes ambientales, es decir, los procesos a escala de paisaje que sustentan a la comunidad o sistema.

Criterios del Rapid Biological Inventories (RBI)

En este trabajo se tienen en cuenta también los criterios de los RBI $[8,9,10,11]$ para la selección de los objetos de conservación y que son los siguientes: $C_{1}$ - tipos de vegetación silvestres 0 hábitats acuáticos que son los fundamentos de la biodiversidad nativa; $\mathrm{C}_{2}$ - tipos de vegetación 0 hábitats acuáticos que son especialmente ricos en especies, diversos 0 amenazados; $\mathrm{C}_{3}-$ comunidades / asociaciones silvestres que son especialmente ricos en especies, diversas 0 abundantes comparadas con las existentes en otros paisajes del país o de la región; $\mathrm{C}_{4}-$ especies, subespecies 0 comunidades / asociaciones que son endémicas del país, de la región $\mathrm{O}$ de la localidad; $\mathrm{C}_{5}$ - comunidades / asociaciones que son raras o están amenazadas, en peligro, vulnerables o en disminución.

Constituyen "objetos de conservación" los elementos con diversidad fisiográfica, biológica, o cultural que conviene conservar en el paisaje y a los que deben dirigirse en este momento la máxima prioridad del manejo $[8,9,10,11]$. A ellos se agregan también las comunidades que realizan funciones irremplazables por otros ecosistemas (ej. los manglares).

A su vez, se consideran "valores para la conservación", los ecosistemas que tienen diversidad de valores a conservar (especies 
endémicas, amenazadas, paraguas, entre otros) y que fundamentalmente tienen poca representatividad o son únicos de la zona estudiada; no obstante, están relativamente poco amenazados por encontrarse en lugares abruptos, sobre lapiés, a gran altitud y/o alejados de áreas pobladas.

La combinación de todos esos criterios [2, 3, 4, 6, $7,8,9,10,11,12]$ constituye la unidad metodológica que será aplicada en este trabajo.

\section{RESULTADOS}

El "valor biológico" (B) de las fitocenosis en esta sierra alcanzó una cifra máxima de 33 y el de "interés para la conservación" (IC) de 44 (Tab. 1).

Las cifras máximas de valor biológico (B) se presentaron en Bosques y matorrales primarios en áreas protegidas de Categoría II (Hohenbergio penduliflorae - Guapiretum obtusatae, entre otros) y en Bosques primarios viables a una altitud mayor de $800 \mathrm{~m}$ snm (Prestoeo acuminatae Ocoteetum, entre otras), mientras las de interés para la conservación (IC) correspondieron a matorrales y bosques del mismo tipo.

De acuerdo con las consideraciones metodológicas de este trabajo, las fitocenosis Prestoeo acuminatae-Ocoteetum, Miconio pterocladae-Magnolietum cubensis y Purdiaeo stenopetalae-Cyrilletum silvae, que fueron estudiadas en el paisaje natural protegido Gran Piedra y en los parques nacionales La Bayamesa y Pico Turquino (con planes operativos, de manejo y con personal para realizarlos, dentro de los que son fundamentales la vigilancia y la protección) no se consideraron amenazadas y por tanto no serán declaradas como objetos de conservación, como sería necesario y recomendable en otras circunstancias. Esto no excluye, sino obliga, el perfeccionamiento, cumplimiento y monitoreo de los planes operativos y de manejo en esas áreas protegidas y la intensificación de la protección de los territorios fuera de dichas áreas.

Por ello, los objetos de conservación debieron circunscribirse a las partes media y baja de la Sierra Maestra, sobre todo a esta última. En dicha parte baja se encontró la mayor parte de la población, tiene a su vez mejores vías de comunicación e instalaciones turísticas y por tanto es donde se produjeron las mayores alteraciones antrópicas y donde los ecosistemas están más amenazados.

En concordancia con esto (Tab. 1), las cinco fitocenosis con un IC mayor de 40 y viables, por estar en un área protegida de Categoría II y/o por encima de los $800 \mathrm{~m}$ snm, no fueron recomendadas como objetos de conservación. A su vez, otras cuatro con un IC menor, de 34.6 a 37 y viables (Pruno-Guareetum guidoneae, Conocarpodo erecti-Coccolobetum uviferae, Oxandro lanceolatae-Burseretum simarubae, Oplonio tetrastichae-Phyllostyletum brasiliensis), por estar en la parte baja de la sierra y altamente amenazadas deben constituirse en objetos de conservación.

Algunas comunidades vegetales de la Sierra Maestra con altos valores de B e IC fueron inviables debido a su pequeño tamaño [Oxandro lanceolatae-Spondiadetum mombin $(\mathrm{B}=26$ e IC = 37), Oplonio tetrastichae-Tabebuietum myrtifoliae (27 y 32.4), Colubrino ellipticae-Gymnanthetum lucidae (27 y 30.4), Tabebuio myrtifoliaeColubrinetum ellipticae (30 y 33), Agavo underwoodii-Plumerietum filifoliae (29 y 32)]. Ellas son sin embargo primarias (ecosistemas relictos y altamente amenazados en la parte baja de la sierra) y únicas de la sierra, incluso locales (con criterios $\mathrm{RBI}-\mathrm{C}_{2}, \mathrm{C}_{4}$ y $\mathrm{C}_{5}$ ), y por esto con gran importancia dentro de este ecosistema de "Escala Nacional o Gruesa". Por ello, todas deben ser consideradas como objetos de conservación, manejadas y protegidas. La primera constituye un relicto del bosque semideciduo mesófilo y las últimas cuatro forman parte del matorral (xeromorfo) costero y precostero, formación vegetal ésta ya considerada anteriormente como objeto de conservación en las Terrazas costeras de la reserva de la biosfera Baconao, puesto que contienen la mayor parte de las especies endémicas (incluso locales), amenazadas, paraguas y funcionales de la parte baja de la sierra.

Tabla 1. Síntesis de la evaluación naturalística, el número promedio de especies por asociación y la viabilidad. Valor biológico $(B)=(N+R+F+U T)-A$, interés para la conservación $(I C)=(B+R C+S-H)$, $\mathrm{Nr}$. sp. = número promedio de especies, viabilidad $(\mathrm{V}=$ viable, $\mathrm{I}=$ inviable $), \mathrm{N}=$ naturalidad, $\mathrm{R}=$ resiliencia, $\mathrm{F}=$ valor florístico-fitocenótico, UT = unicidad fitocenótica, $A=$ amenazas al ecosistema, $\mathrm{RC}=$ retención de carbono, $\mathrm{S}-\mathrm{H}=$ 
protección del suelo y mantenimiento de la calidad de

7) -2

\begin{tabular}{|c|c|c|}
\hline $\begin{array}{l}\mathrm{B}(\mathrm{N}, \\
\mathrm{R}, \mathrm{F}, \\
\text { UT) - }\end{array}$ & Fitocenosis & $\begin{array}{c}\mathrm{IC}(\mathrm{B}, \\
\mathrm{RC}, \\
\mathrm{SH})\end{array}$ \\
\hline
\end{tabular}

las

cubensis

Purdiaeo

stenopetalae -

Cyrilletum silvae aguas.

$$
\begin{array}{cc}
41(2, & 41.4 \\
7) & \mathrm{C}_{4}
\end{array}
$$

Bosques primarios viables con más de 34 de IC fuera de Comunidades inviables a una altitud mayor de $800 \mathrm{~m} \mathrm{snm}$ áreas protegidas

26 Oplonio tetrastichae 37 (2

(10, - Phyllostyletum

8, $9, \quad$ brasiliensis

6) -7

25

$(10$

8,8 ,

6) -7

24

$(10$,

8,8 ,

5) -7

24

$(10$,

8,7 ,

6) -7

Conocarpodo erecti

- Coccolobetum

uviferae

Oxandro

lanceolatae -

9) $\quad \mathrm{C}_{4}$

$\mathrm{C}_{4}$

$36(2, \quad 32.3 \quad \vee \quad 7)-2$

9) $\quad \mathrm{C}_{4}$

simarubae

Pruno-Guareetum

guidoneae

$35(2$,

57

9) $\quad \mathrm{C}_{4}$

Bosques y matorrales primarios en áreas protegidas Categoría II (RE - Pozo Prieto)

$\begin{array}{cccc}32 & \text { Hohenbergio } & 43(2, & 60.5 \\ (10, & \text { penduliflorae - } & 9) & \mathrm{C}_{4} \\ 8,9, & \text { Guapiretum } & & \\ 7)-2 & \text { obtusatae } & & \\ 32 & \text { Ocoteo coriaceae - } & 43(2, & 36.8 \\ (10, & \text { Guapiretum } & 9) & \mathrm{C}_{4} \\ 8,9, & \text { obtusatae } & & \end{array}$

7) - 2

32

$(10$,

Rhipsali cassuthae

- Hohenbergietum

$34(1, \quad 38.1$

$34.6 \quad 3.8$

(1.6, $\quad \mathrm{C}_{4}$

3.8
$\mathrm{C}_{4}$

V $14(2$,

8, 9, penduliflorae

7) - 2

$17(5$,

4,4 ,

7) - 3

$13(2$,

4,3 ,

1)

$\mathrm{C}_{4}$

$\mathrm{C}_{4}$

V $13(1$,

$\mathrm{V} 1,3$,

V 6) - 2

Guareo guidoniae - $22.4 \quad 54$

Dendropanacetum

(1.4,

$\mathrm{C}_{4}$

4)

Ocoteo coriaceae -

Alvaradoetum

$18.4 \quad 51$

(1.4, $\mathrm{C}_{4}$
$26(7$,

7,7 ,

7) - 2

$24(7$,

5,7 ,

$17(5$

$17(5$,

4, 4 ,

6) - 2

$22(9$,

1,6 ,

7) -2

4, 3,

7) -2

$14(1$,

2, 6 ,

7) - 2

2, 6 ,

6) -2

$11(1$,

6) - 2

$9(1$,

1,3 ,

7 (1,

2, 3,

3) -2

Prestoeo

acuminatae -

Syzygietum jambi

Eugenio

scaphophyllae -

Clusietum

tetrastigmae

Cyatheo -

Cyrilletum silvae

Cypero lanceolati -

Cyrilletum silvae

Morello ceriferae -

Cyrilletum silvae

Blechnetum lineati

$34.4 \quad 30.5$

(1.4, $\mathrm{C}_{4}$

7)

$32.4 \quad 46.5$

(1.4,

7)

$25(1, \quad 23.8$,

7) $\quad 33.3$

I (C)

$25\left(1, \quad \begin{array}{cc}\mathrm{C}_{4} \\ \mathrm{C} & 17.5\end{array}\right.$

2) $\quad \mathrm{C}_{4}$

I $(\mathrm{T}, \mathrm{C})$

$22.2 \quad 43$

(1.2, $\quad \mathrm{C}_{4}$

7)

$19(1, \quad 15.3 \quad \mathrm{I}(\mathrm{T}, \mathrm{C})$

4) $\quad \mathrm{C}_{4}$

Dicranopterido

flexuosae -

Cyrilletum silvae

Piperi adunci -

Myrsinietum

coriaceae

Ageratino

paucibracteatae -

Cyrilletum silvae

Andropogono-

Pteridietum

arachnoidei

Gleichenello

pectinatae

$19(1, \quad 14$

I $(\mathrm{T}, \mathrm{C})$

5) $\quad \mathrm{C}_{4}$

$16(1, \quad 31,46 \quad$ I $(\mathrm{T}, \mathrm{C})$

4) $\quad \mathrm{C}_{4}$

$14(1, \quad 26 \quad \mathrm{I}(\mathrm{T}, \mathrm{C})$

4) $\quad \mathrm{C}_{4}$

$12(1, \quad 28$

4) $\mathrm{C}_{4}$

$19(1, \quad 16.5 \quad \mathrm{I}(\mathrm{T} ; \mathrm{C})$

5)

6) -2

7) - 3 arborescentis

Bosques primarios viables a una altitud mayor de $800 \mathrm{~m} 8,7$, snm

32 Prestoeo

$(10, \quad$ acuminatae -

8, 9, Ocoteetum

7) - 2

32

$(10$

8,9 ,
Miconio

pterocladae -

Magnolietum

$43(2, \quad 41.3$

9) $\quad \mathrm{C}_{4}$

$41(2, \quad 48.6$

7) $\quad \mathrm{C}_{4}$
3) -7

$21(8$,

7,8 ,

5) -7

$20(7$,

V 7,8,

5) -7

20 (7,
Comunidades viables áreas protegidas

Cupanio glabrae -

Ocoteetum coriaceae

Trichilio hirtae -

Guazumetum ulmifoliae

Sideroxyletum

salicifolio -

foetidissimi

Canello winteranae
(1.6,

9)

29.6

(1.6,

28.6

(1.6,

7)

27.6

51

$\mathrm{C}_{4}$

33.1

46

$\mathrm{C}_{4}$

40.5,
V

V

V

$\sqrt{2}$

V 


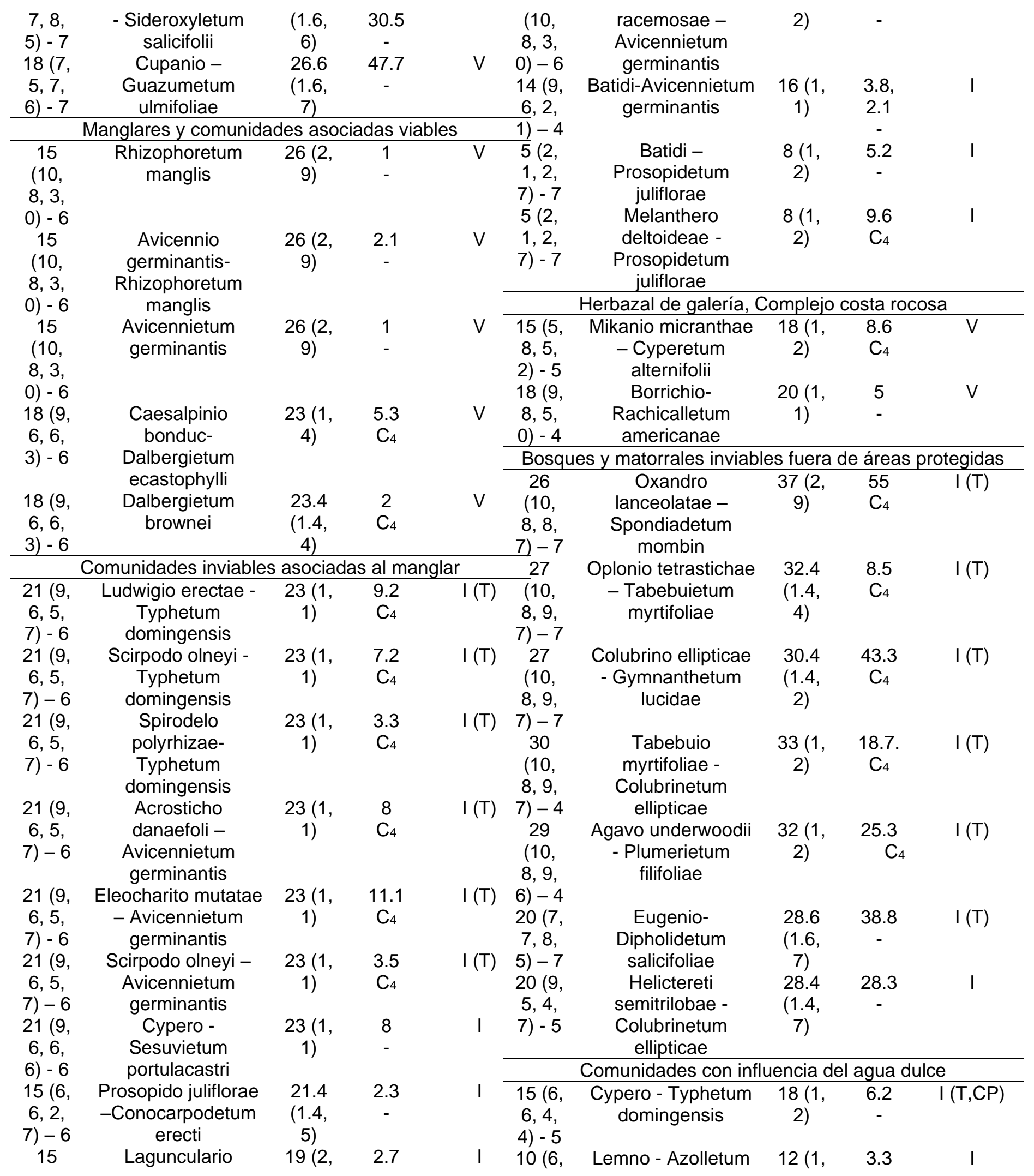




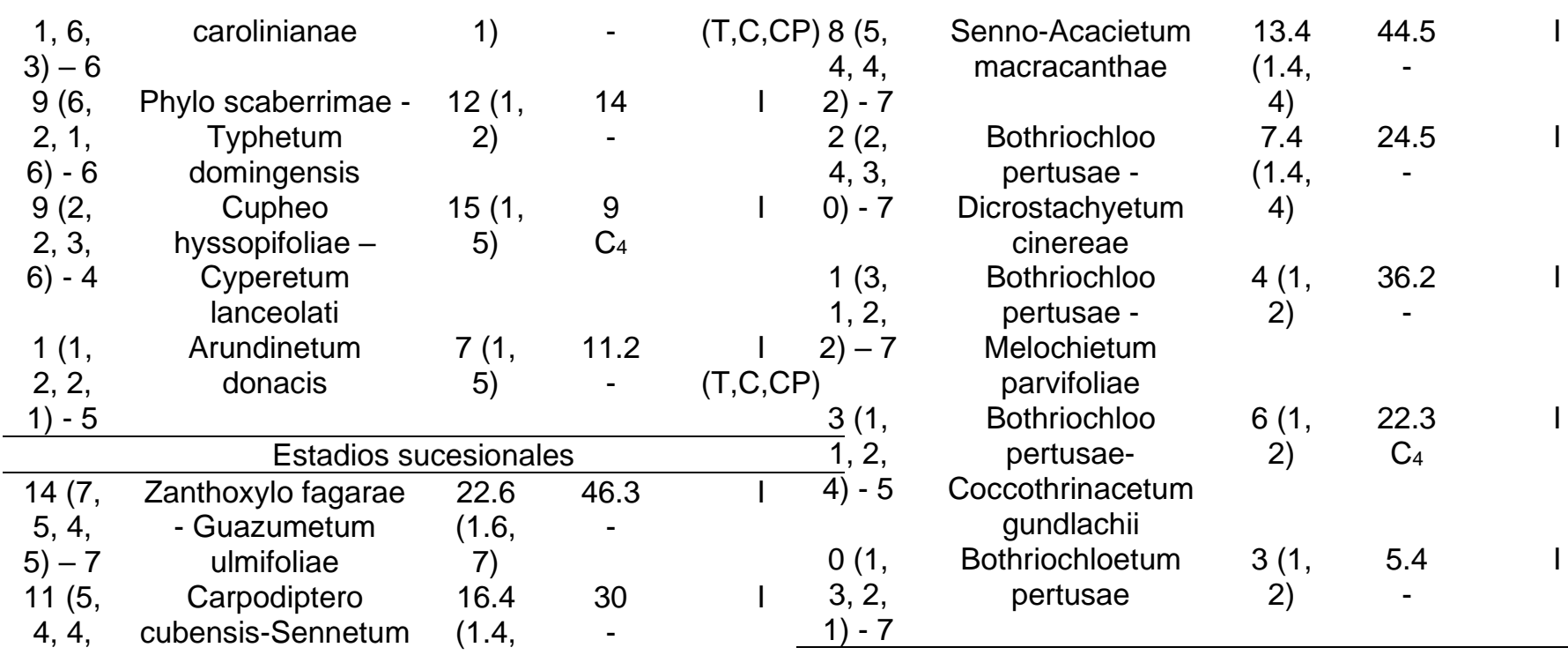

5) - $7 \quad$ atomariae

4)

$11(5, \quad$ Varronio globosae- $\quad 16.4$

4, 4, Sennetum (1.4

13.6

I

5) -7 atomariae

11 (5, Varronio globosae- 16.4

4, 4, Acacietum (1.4,

$4)$
16.4
4.4

Al comparar la composición florística de estas fitocenosis con la de las formaciones vegetales a las que corresponden, se observó que estas últimas tienen una importancia similar que sus fitocenosis respecto a los aspectos de interés para la conservación (endemismo, especies paraguas y amenazadas, unicidad, paisaje). Por ello se consideró extender la importancia para la conservación (objetos de conservación, valores para la conservación) de estas fitocenosis a las formaciones vegetales, dado a que estas tienen mayor importancia práctica y son fácilmente reconocibles (lo que es difícil de las fitocenosis en particular) por el personal dedicado a la conservación, los decisores y la población local.

Se apreció, que las seis fitocenosis objetos de conservación viables y con mayor valor de IC y las cinco inviables con igual carácter se corresponden con el: bosque siempreverde mesófilo $\left(\mathrm{C}_{5}\right)$, bosque siempreverde micrófilo $\left(\mathrm{C}_{2}\right)$, bosque semideciduo mesófilo $\left(\mathrm{C}_{3}\right)$, bosque semideciduo micrófilo $\left(\mathrm{C}_{2}\right)$, matorral (xeromorfo) costero y precostero $\left(\mathrm{C}_{2}\right)$ y uveral $\left(\mathrm{C}_{1}\right)$, formaciones vegetales éstas que se convirtieron a su vez en objetos de conservación.

Debido al inminente efecto del cambio climático, las autoridades cubanas han decidido que el

ecosistema de manglar en todo el archipiélago cubano tenga interés prioritario para la conservación, por lo que las asociaciones viables Rhizophoretum manglis (IC = 26), Avicennio germinantis-Rhizophoretum manglis (26), Avicennietum germinantis (26), Caesalpinio bonduc-Dalbergietum ecastophylli (23), Dalbergietum brownei (23.4) y otras inviables dentro del mismo ecosistema fueron consideradas como el objeto de conservación "manglares" $\left(C_{1}\right)$.

\section{Amenazas (tensores) principales, fundamentalmente en la parte baja de la Sierra Maestra}

Las principales amenazas sobre los ecosistemas en la parte baja (fundamentalmente) de la Sierra Maestra fueron:

- Carretera y caminos que destruyeron, fragmentaron $y / 0$ alteraron los ecosistemas y facilitaron el movimiento descontrolado de las personas para realizar actividades que afectan el ambiente, incluido el turismo sin regulación.

- Incumplimiento del marco regulatorio ambiental cubano, es decir, se produjeron actividades humanas que provocaron 
fragmentación y/o gran alteración a los ecosistemas naturales (ej. corta de madera para leña, carbón, extracción furtiva de maderas preciosas para las artesanías y otros usos), alteración de la vegetación por animales domésticos sueltos, incendios antrópicos, degradación de los hábitats por el pastoreo extensivo y los cultivos transitorios, sustracción de arena y piedras y construcción de instalaciones turísticas.

- Plantas y animales exóticos invasores y ruderales, los que produjeron una gran presión sobre los elementos naturales.

- Perturbaciones catastróficas como huracanes, sequías intensas y erosión acelerada que pueden eliminar ecosistemas y/o poblaciones.

- Aumento del nivel medio del mar y de la temperatura que pueden inundar áreas destruyendo hábitats y modificar la composición florística de los ecosistemas cliseriales.

- Particularizando los manglares, éstos se afectaron por: extracción de corteza y fustes, destrucción para áreas agrícolas, vertimientos de residuales sólidos y líquidos, insuficiente conocimiento de las herramientas del manejo integrado costero e intervenciones incontroladas.

\section{DISCUSION}

En concordancia con la planificación ecorregional, la Sierra Maestra (Fig. 1) es un área que es importante conservar porque contiene plantas, animales, formaciones vegetales, fitocenosis de diversa categoría, paisajes y sistemas ecológicos únicos territorialmente (incluso por distritos fitogeográficos) y viables. En consonancia con ello, presenta las condiciones para constituirse en un "Área de conservación funcional" u "Objeto de conservación de filtro grueso" $[6,7]$ y/o "Nacional o Gruesa" [12].

Las nueve fitocenosis viables con más de 34 de IC son bosques primarios, de ellas hay seis que tipifican los pisos altitudinales observados en la Sierra Maestra. Ellas representan a su vez las formaciones vegetales básicas del "geosigmetum cliserial" de este macizo montañoso.
Figura 1. Situación geográfica de la Sierra Maestra y sus principales fajas altitudinales de interés conservacionista.

La parte alta de la sierra (por encima de los $800 \mathrm{~m}$ snm) tiene una extraordinaria importancia hidrológica, pues en ella hay una alta pluviosidad [15]; al mismo tiempo aquí se produce la condensación casi diaria de las nieblas y nubes bajas, las que [16] representan alrededor del $50 \%$ de las lluvias y que [17] tienen gran importancia hídrica; además la evaporación es baja y la insolación relativa es generalmente menor del 20 $\%$ [18]; todo ello contribuye altamente a un balance hidrológico favorable. En esa zona los bosques tienen una conspicua estera radical, la que funciona como una esponja, absorbiendo el agua de la condensación; ésta, el follaje y el enraizamiento superficial inducen la infiltración de las precipitaciones, convirtiéndose en un excepcional reservorio hídrico; por ello, de aquí salen los ríos y arroyos importantes. Los suelos ferralíticos característicos del área son muy erosionables cuando están descubiertos, pero altamente resistentes a dicha erosión cuando están cubiertos por la vegetación.

En esa zona, la que constituye los distritos fitogeográficos Sierra del Turquino (28) y Gran Piedra (29), se concentra el gran endemismo fanerogámico $[19,20,21]$ y la mayor parte de los musgos, hepáticas y helechos presentes en la sierra [22, 23, 24, 25], es además muy rica en líquenes [26]. Aquí se presentan también la mayor parte de las especies amenazadas presentes en la Sierra Maestra, tanto de las hepáticas, musgos y helechos, como de las fanerógamas $[27,28,29$, 30]. Al mismo tiempo, gran parte de esos territorios constituyen áreas protegidas (parques nacionales: La Bayamesa y Pico Turquino, reservas ecológicas: Pico Caracas y Pico Mogote, reserva florística manejada Loma del Gato-Monte Líbano, paisaje natural protegido Gran Piedra). Incluso, se recomendaron como objetos de conservación [31, 32, 33] en el parque nacional La Bayamesa y la reserva ecológica Pico Mogote a "la pluvisilva montana y sus estadios sucesionales, el bosque nublado, el matorral nublado natural y los relictos de pinares naturales" ya que consideraron que dichas formaciones vegetales eran vitales para la conservación de la fitodiversidad de la Sierra Maestra. A su vez, se 
recomendó [34] que esta zona debía tratarse muy cuidadosamente sin transformaciones importantes. Por ello, ese territorio que se corresponde con los pisos altitudinales de pluvisilvas y bosques nublados de la Sierra Maestra (más de $800 \mathrm{~m} \mathrm{snm}$ ), fueron declarados en las "Metas Nacionales para la Diversidad Biológica", como "protectoras para la biodiversidad, aguas y suelos" [35].

Importante para la preservación ecosistémica es el conocimiento obtenido por diversos estudios florísticos [30, 31, 36, 37, 38, 39, 40, 41, 42, 43, $44,45,46,47,48,49,50]$ sobre la Sierra Maestra (endemismo, especies paraguas y amenazadas, unicidad, paisaje, entre otros), lo que ratifica la valoración expuesta de las asociaciones (para la práctica) y las formaciones vegetales como los objetos a evaluar respecto a la conservación.

Por ello, las siete formaciones vegetales expuestas como objetos de conservación son los elementos prioritarios para los planes de manejo y operativos. A ellas deben dirigirse los esfuerzos de manejo y protección, tanto institucionales como de la población, de educación ambiental y vigilancia.

De esas siete formaciones vegetales objetos de conservación, cinco corresponden a la parte baja de la sierra, incluso cuatro se incluyen en la franja costera y precostera. Ésta constituye a su vez, el área más amenazada y con mayores dificultades de manejo, con áreas importantes fuera de áreas protegidas, con población dispersa y/o concentrada en todo el litoral y cercana a las áreas con valores para la conservación y por tanto el principal problema conservacionista de la Sierra Maestra. En esta zona se encuentra la mayor cantidad de poblados (Pilón, Mota, Ocujal, Uvero, Chivirico, Aserradero, Siboney, Juraguá, Sigua, Baconao, entre otros), diversas áreas con población dispersa, una parte cercana a la ciudad de Santiago de Cuba, con varias instalaciones turísticas nacionales e internacionales (Baconao, Sevilla, Mota), carreteras desde Pilón a Baconao y playas con gran afluencia de público. Todas ellas impactan de disímiles formas sobre esta zona. En ella Figueredo [51] consideró al manglar, el bosque semideciduo micrófilo y el matorral (xeromorfo) costero y precostero, como objetos de conservación para la reserva de la biosfera Baconao.

También se identificó la deforestación, los incendios forestales, la extracción de especies para diversos usos, el pastoreo extensivo y la fabricación de carbón, como amenazas fundamentales en estas terrazas costeras [51]; aspectos éstos también reconocidos para Latinoamérica [52]. Para esta área el turismo se considera una amenaza directa, fundamentalmente por la construcción de instalaciones y la transformación de áreas, lo cual coincide con lo anteriormente registrado [53] para el país y la Estrategia Nacional Ambiental [54].

\section{CONCLUSIONES}

La combinación de la evaluación naturalística (modificada), la viabilidad y los criterios del Rapid Biological Inventories constituye una unidad metodológica eficiente para valorar la conservación ecosistémica en Cuba.

Las áreas por encima de los $800 \mathrm{~m}$ snm deben priorizarse como "protectoras para la biodiversidad, las aguas y los suelos".

Las medidas de manejo y protección deben concentrarse en las zonas costeras y precosteras de la Sierra Maestra, pues constituyen las áreas más amenazadas.

Las asociaciones con mayor valor de IC y con criterios $\mathrm{RBI}$, se encuentran en las formaciones vegetales siguientes: bosque siempreverde mesófilo, bosque siempreverde micrófilo, bosque semideciduo mesófilo, bosque semideciduo micrófilo, matorral (xeromorfo) costero y precostero, manglar y uveral, las que se convierten en objetos de conservación.

\section{REFERENCIAS BIBLIOGRÁFICAS}

[1] CIGEA. Centro de Información, Gestión y Educación Ambiental. Estrategia Ambiental Nacional de Cuba 2010 - 2013. Agencia de Medio Ambiente. CITMA. 2010.

[2] Loidi, J. Phytosociology applied to nature conservation and land management. En: Song, Y., Dierschke, H. \& Wang, X. (Eds.). Proc. 36 th IAVS Symp. Shanghai. East China Normal Univ. Press. 1994.

[3] Loidi, J. La Fitosociología como elemento renovador de la Botánica española en la segunda mitad del siglo XX. Lazaroa 25: 15-21. 2004.

[4] Loidi, J. La Fitosociología como proveedora de herramientas de gestión. Lazaroa 29: 7-17. 2008. 
[5] Reyes, O.J. Delta del río Cauto, Segundo Humedal del Caribe Insular, Cuba. Vegetación y Sintáxones. Editorial Academia Española. 2014.

[6] Craig, G., L. Valutis, D. Vosick, B. Neerly, K. Wheaton, J. Touval \& G. Rumels. Diseño de una Geografía de la Esperanza: Manual para la planificación de la conservación ecorregional. Segunda Edición Vol. I. Manual para el Planificador. The Nature Conservancy. 2000 a.

[7] Craig, G., L. Valutis, D. Vosick, B. Neerly, K. Wheaton, J. Touval \& G. Rumels. Diseño de una Geografía de la Esperanza: Manual para la planificación de la conservación ecorregional. Segunda Edición Vol. II. Manual para el Planificador. The Nature Conservancy. 2000 b.

[8] Fong, A., D. Maceira, W.S. Alverson \& T. Wachter (Eds.). Cuba: Parque Nacional Alejandro de Humboldt. En: Rapid Biological Inventories 14. The Field Museum, Chicago, IIIn. 2005 a.

[9] Fong, A., Maceira, D., Alverson, W.S. \& Shopland, J.M. (Eds.). Objetos de conservación. hj[14] Capote-Fuentes, R.T. Resiliencia de los manglares asociados al río Santa Ana, La Habana, Ciudad de La Habana, Cuba. [Tesis M.Sc.]. Instituto Ecología Sistemática, CITMA. 2003.

[15] Trusov, I.I., Izquierdo, A. \& Díaz, L.R.. Características espaciales y temporales de las precipitaciones atmosféricas en Cuba. Academia Ciencias Cuba, La Habana, Instituto de Geografía. 1983.

[16] Samek, V. \& A. Travieso. Climaregiones de Cuba. Rev. Agricultura 2: 5-23. 1968.

[17] Boytel Yambú, F. Geografía eólica de Oriente. Inst. Cubano del Libro. 1972.

[18] Montenegro, U. Insolación relativa. En: Atlas de Santiago de Cuba. Academia de Ciencias de Cuba, Santiago de Cuba. Mapa 26. 1991.

[19] Samek, V. Pinares de la Sierra de Nipe; Estudio Sinecológico. Academia Ciencias Cuba, La Habana. Serie Forestal 14. 1973.

[20] Borhidi, A. Phytogeography and Vegetation Ecology of Cuba. Akadémiai Kiadó. Budapest. 1991.

[21] Fagilde M.C. El endemismo en la Sierra Maestra. En Diversidad biológica del macizo montañoso Sierra Maestra. Centro Oriental de Ecosistemas y Biodiversidad (BIOECO).
Riesgos y oportunidades para la conservación. En: Rapid Biological Inventories. Report 10. Cuba: Siboney-Juticí. The Field Museum, Chicago IIIn. 37-38. 2005 b.

[10] Maceira, D., Fong, A., Alverson, W.S. \& Wachter, T. (Eds.). Cuba: Parque Nacional La Bayamesa. En: Rapid Biological Inventories 13. The Field Museum, Chicago, Illn. 2005.

[11] Maceira, D., Fong, A. \& Alverson, W.S. (Eds.) Cuba: Pico Mogote. En: Rapid Biological Inventories 09. The Field Museum, Chicago, IIIn. 2006.

[12] Gerhartz, J.L., Estrada, R., Hernández, E., Hernández, A. \& González, A. Metodología para la elaboración de Planes de Manejo en Áreas Protegidas de Cuba. SNAP. Editorial Feijóo. Univ. Central Las Villas. 2008.

[13] Reyes, O.J. \& F. Acosta. Fitocenosis en las pluvisilvas sobre ofiolitas del Parque Nacional Alejandro de Humboldt, Cuba Oriental. Caldasia 3 (1): 91-123. 2017.

Programa C.T. Nacional Desarrollo Sostenible de la Montaña. Tomo I. 427-436. 2000.

[22] Potrony, M.E. Inventario y distribución de los musgos de la Sierra Maestra, Cuba. [Tesis M. Sc.]. Centro Oriental de Ecosistemas y Biodiversidad (BIOECO), CITMA. 1999.

[23] Caluff, M.G. \& Shelton, G. 1998. Helechos y plantas afines. En: Diversidad biológica del Macizo Montañoso Nipe-Sagua-Baracoa. Programa C.T. Nacional Desarrollo Sostenible de la Montaña. Centro Oriental de Ecosistemas y Biodiversidad (BIOECO). Vol. III. pp. 450-473.

[24] Mustelier, K. Hepáticas y antoceros. En: Diversidad biológica de los macizos de Cuba Oriental. (Eds. Viña, N., Fong, A. y Maceira, D.).Tomo 2, 2001-2006. Centro Oriental de Ecosistemas y Biodiversidad (BIOECO), Santiago de Cuba. 2001.

[25] Motito, A. Los musgos de Cuba Oriental; aspectos sobre su distribución, ecología y conservación. [Tesis Dr. C.]. Centro Oriental de Ecosistemas y Biodiversidad. 2003.

[26] Rosabal, D., Burgaz, A.R. \& Reyes, O.J. Diversidad y distribución vertical de líquenes corticícolas en la pluvisilva montana de la Gran Piedra, Cuba. Botánica Complutensis 36: 19-30. 2012. 
[27] Mustelier, K., Trapero, A. \& Vicario, A. Hepáticas y anthoceros amenazados en Sierra Maestra. En: Diversidad biológica de macizo montañoso Sierra Maestra. (Eds. Viña, N., Fong, A. y Maceira, D.). Tomo I: 232-235. 2000.

[28] Motito, A. \& Potrony, M.E. Musgos amenazados en la Sierra Maestra. En: Diversidad biológica de macizo montañoso Sierra Maestra. (Eds. Viña, N., Fong, A. y Maceira, D.). Tomo I. Centro Oriental de Ecosistemas y Biodiversidad (BIOECO). 236-242. 2000.

[29] Caluff, M.G. \& Shelton, G. Helechos y plantas afines amenazadas en la Sierra Maestra. En: Diversidad biológica del macizo montañoso Sierra Maestra. (Eds. Viña, N., Fong, A. y Maceira, D.). Tomo I: 243-246. 2000.

[30] Echezarreta, C., D. Duany, O. Pacheco, M. Gelis, A. Pacheco, A. Hernández, C. Martínez \& F. Polanco. Fanerógamas amenazadas en Sierra Maestra. En: Diversidad biológica del macizo montañoso Sierra Maestra. Centro Oriental de Ecosistemas y Biodiversidad (BIOECO). Programa C.T. Nacional Desarrollo Sostenible de la Montaña. Tomo I: 247-255. 2000.

[31] Reyes, O.J. \& Acosta, F. Vegetación. Cuba: Parque Nacional La Bayamesa. En: Rapid Biological Inventories: 13. (Eds. Maceira, D., A. Fong, W.S. Alverson y T. Wachter). The Field Museum, Chicago. 43-50. 2005 a.

[32] Reyes, O.J. \& Acosta, F. Vegetación terrestre. En: Rapid Biological Inventories. Report 10. Cuba: Siboney-Juticí. 46-50. (Eds. Fong, A., Maceira, D., Alverson, W.S. \& Shopland, J.M.). The Field Museum, Chicago. 2005 b.

[33] Reyes, O.J. \& Acosta, F. Vegetación. Pico Mogote. En: Rapid Biological Inventories. Report 09. (Eds. Maceira, D., A. Fong \& W.S. Alverson). 40-46. The Field Museum, Chicago, IIIn. 2006.

[34] Álvarez, P.A. \& Varona, J.O. Silvicultura. La Habana. Ed. Pueblo y Educación. 1988.

[35] PNUD/GEF. Cuba, metas nacionales para la diversidad biológica 2016-2020. Proyecto PNUD/GEF "Plan Nacional de Diversidad Biológica para apoyar la implementación del Plan Estratégico del CDB 2011 - 2020 en la República de Cuba". 31. 2016.

[36] Duany, D. \& C. Mariño. Estudio de la composición florística de la vegetación espinar tropical de la Estación de la Reserva Natural "El
Retiro". [Trabajo de Curso]. Universidad de Oriente. Santiago de Cuba. 1977.

[37] Bermúdez, F. Contribución al Conocimiento Florístico de la Vegetación Xeromorfa Costera y Subcostera de Siboney - Justici - Sardinero, desde 1973-1984. Documentos del Centro Oriental de Ecosistemas y Biodiversidad (BIOECO). 1984.

[38] Castilla, R., R. Menéndez, R. Berazaín, L.A. Kuznetzov, O. Pelicié, D. Reyes \& M. Rodríguez. Comparación de la flora y la vegetación de dos zonas xerófitas del Parque Baconao. Memorias del Primer Simposio de Botánica. Tomo III. 99103. 1985.

[39] Menéndez, R., Castilla, R., Pelicié, O., Reyes, D. \& Rodríguez, M. Ecología de las maniguas xerofíticas costeras del Parque Baconao. Instituto Superior Pedagógico "Frank País". 11-16. 1985.

[40] Menéndez, R., Castilla, R., Pelicié, O., Reyes, D., Berazaín, R. \& Kuznetzov, L.A. Introducción al estudio de la vegetación y la flora de la parte costera de la región de Baconao. Rev. Jardín Botánico Nacional 7(1): 37-47. 1986.

[41] Capote, R.P., N. Ricardo, D. Vilamajó, R. Oviedo \& E.E. García. Flora y vegetación de la zona costera entre Daiquiri y Verraco, Parque Baconao, Santiago de Cuba. Acta Botánica Cubana. No.48. Academia de Ciencias de Cuba. 1987.

[42] Reyes, O.J., Acosta, F., Oviedo, R. \& Bermúdez, F. La Reserva Florística Manejada Justicí y sus alrededores, notas sobre su flora y vegetación. Biodiversidad de Cuba Oriental, Editorial Academia. Vol. III. 12-23. 1999.

[43] Figueredo, L.M., A. Salmerón, A., Álvarez, L.O. \& Acosta, G. Problemática ambiental y acciones para la Reserva Ecológica Siboney. Foresta Veracruzana. 1(2): 41-46. 1999.

[44] Figueredo, L.M., Acosta, F., Reyes, O.J. \& Fornaris, E. Caracterización de la vegetación de las Terrazas Costeras de la Reserva de la Biosfera Baconao, Santiago de Cuba, Cuba. Brenesia 78: 25-33. 2012.

[45] Bermúdez, F., Figueredo, L.M. \& González, A. La Reserva Ecológica Siboney-Juticí. Informe de Proyecto (Inédito). Centro Oriental de Ecosistemas y Biodiversidad. Santiago de Cuba. 2001a. 
[46] Bermúdez, F., Figueredo, L.M., Reyes, O.J., González, A. \& Viña Dávila, L. Estudio florístico de la Reserva Ecológica Siboney-Juticí. Documentos del Centro Oriental de Ecosistemas y Biodiversidad (BIOECO). 2001b.

[47] Martinez Quesada, E. \& Fagilde, M.C. Fanerógamas. En: Estudio y manejo del Paisaje Natural Protegido Gran Piedra. Informe de Proyecto. Centro Oriental de Ecosistemas y Biodiversidad (BIOECO), Santiago de Cuba. 6-11. 2001.

[48] Martínez Quesada, E. \& Alverson, W.S. Plantas vasculares terrestres. En: Rapid Biological Inventories. Report 10. Cuba: Siboney-Juticí. Fong, A., Maceira, D., Alverson, W.S. \& Shopland, J.M. (Eds.). The Field Museum, Chicago. 52. 2005.

[49] Martínez Quesada, E., Reyes, O.J., Viña, N., Viña, L., Pacheco, O., Acosta, F. \& Ferret, H. Características ecológicas y poblacionales de Melocactus nagyii y $M$. evae en Cuba. Foresta Veracruzana 7(1): 25-30. 2009.
[50] Acosta-Costa, J., Castell-Puchades, M.A. \& Álvarez-Quintana, L.O. Caracterización de los bosques semideciduos mesófilo y micrófilo en el Refugio de Fauna El Macío, Granma, Cuba. Ciencia en su PC Nr. 2, abril-junio. 26 pp. 2014.

[51] Figueredo Cardona, L.M. Prioridades para la conservación de la flora y la vegetación de los Cerros Calizos Costeros de la Reserva de la Biosfera Baconao. [Tesis M.Sc.]. Instituto de Ecología y Sistemática, CITMA. 2009.

[52] Primack, R., R. Rozzi, P. Feisinger, R. Dirzo \& F. Massardo. Fundamentos de Conservación Biológica Perspectivas Latinoamericanas. Fondo de Cultura Económica, México D. F. 2001.

[53] Vales, M., Álvarez, A., Montes, L. \& Ávila, A. (comp.). Estudio Nacional sobre la Diversidad Biológica en la República de Cuba. PNUMA, CenBio, IES, AMA, CITMA. La Habana. CESYTA, Madrid. 1998.

[54] CIGEA. Centro de Información, Gestión y Educación Ambiental. Estrategia Ambiental Nacional de Cuba. CIGEA, Agencia de Medio Ambiente, CITMA. 2007. 